Pacific

Journal of

Mathematics

QUOTIENTS OF MILNOR $K$-RINGS, ORDERINGS, AND VALUATIONS

IDO EFRAT 


\title{
QUOTIENTS OF MILNOR $K$-RINGS, ORDERINGS, AND VALUATIONS
}

\author{
IDO EFRAT
}

\begin{abstract}
We define and study the Milnor $K$-ring of a field $\boldsymbol{F}$ modulo a subgroup of the multiplicative group of $F$. We compute it in several arithmetical situations, and study the reflection of orderings and valuations in this ring.
\end{abstract}

\section{Introduction}

Let $F$ be a field and let $F^{\times}$be its multiplicative group. The Milnor $K$-ring $K_{*}^{M}(F)$ of $F$ is the tensor (graded) algebra of the $\mathbb{Z}$-module $F^{\times}$modulo the homogeneous ideal generated by all elements $a_{1} \otimes \cdots \otimes a_{r}$, where $1=a_{i}+a_{j}$ for some $1 \leq$ $i<j \leq r$ [Milnor 1970]. Alongside with $K_{*}^{M}(F)$, the quotients $K_{*}^{M}(F) / m=$ $K_{*}^{M}(F) / m K_{*}^{M}(F)$, for $m$ a positive integer, also play an important role in many arithmetical questions. In this paper we study a natural generalization of these two functors. Specifically, we consider a subgroup $S$ of $F^{\times}$and define the graded ring $K_{*}^{M}(F) / S$ to be the quotient of the tensor algebra over $F^{\times} / S$ modulo the homogeneous ideal generated by all elements $a_{1} S \otimes \cdots \otimes a_{r} S$, where $1 \in a_{i} S+a_{j} S$ for some $1 \leq i<j \leq r$. The graded rings $K_{*}^{M}(F)$ and $K_{*}^{M}(F) / m$ then correspond to $S=\{1\}$ and $S=\left(F^{\times}\right)^{m}$, respectively.

The ring-theoretic structure of $K_{*}^{M}(F) / S$ reflects many of the main arithmetical properties of $F$, especially those related to orderings and valuations. We illustrate this by computing it in the following situations:

(1) $F^{\times} / S$ is a finite cyclic group. Here, if $F$ has no orderings containing $S$ then $K_{*}^{M}(F) / S$ is trivial in degrees $>1$. Otherwise $K_{*}^{M}(F) / S$ coincides in degrees $>1$ with the tensor algebra over $\{ \pm 1\}$ (Theorem 4.1). This includes as a special case the computation of the Milnor $K$-ring of finite fields, which goes back to Steinberg and Milnor [Milnor 1970, Example 1.5].

(2) There is a (Krull) valuation $v$ on $F$ whose 1-units are contained in $S$. We show that under a mild assumption, $K_{*}^{M}(F) / S$ is then obtained from the corresponding $K$-ring of the residue field and from $v\left(F^{\times}\right) / v(S)$ by means of a

MSC2000: primary 19F99; secondary 12J10, 12J15, $12 \mathrm{E} 30$.

Keywords: generalized Milnor $K$-rings, orderings, valuations.

The research has been supported by the Israel Science Foundation grant No. 8008/02-1. 
natural algebraic construction analogous to the construction of a polynomial ring over a given ring (Section 5).

(3) $F^{\times} / S$ is finitely generated, and is generated by the 1 -units of a rank-1 valuation $v$ such that $S$ is open in the $v$-topology on $F$. We then prove that $K_{*}^{M}(F) / S$ is trivial in degrees $>1$ (Theorem 6.2).

(4) $F^{\times} / S$ is finite, and there is a rank-1 valuation $v$ on $F$ with mixed characteristics $(0, p)$ such that $S=\left(F^{\times}\right)^{p}\left(1+p^{2} \mathfrak{m}_{v}\right)$, where $\mathfrak{m}_{v}$ is the valuation ideal (when $v$ is Henselian the latter condition just means that $S=\left(F^{\times}\right)^{p}$ ). We show that then $K_{*}^{M}(F) / S$ is either the Milnor $K$-ring of a finite extension of $\mathbb{Q}_{p}$, or else it is trivial in degrees $>1$ and $v\left(F^{\times}\right)$is $p$-divisible (Theorem 7.4). The proof is based on the vanishing theorem of (3) above.

These results are mostly of a local nature. In a forthcoming paper we compute the functor $K_{*}^{M}(F) / S$ in global situations, where $S$ is related to a family of orderings and valuations.

Studying Milnor's $K$-theory modulo a subgroup $S$ by means of the functor $K_{*}^{M}(F) / S$ resembles the reduced theory of quadratic forms: there one studies quadratic forms modulo a preordering $T$ on $F$ via the reduced Witt ring functor $W_{T}(F)$, rather than the classical Witt ring; see [Lam 1983; Becker and Köpping 1977] for details.

Furthermore, the celebrated Bloch-Kato-Milnor conjecture predicts that $K_{*}^{M}(F)$ is isomorphic to the Galois cohomology of the absolute Galois group $G_{F}$ of $F$ with respect to twisted cyclotomic actions [Kahn 1997]. Similarly, when $p$ is a prime number and $F$ contains a primitive $p$-th root of unity, $K_{*}^{M}(F) / p$ is related to the Galois cohomology ring of the maximal pro- $p$ Galois group $G_{F}(p)$ of $F$ with its trivial action on $\mathbb{Z} / p$. From this viewpoint, the generalized functor $K_{*}^{M}(F) / S$ serves in some sense as an analog of the Galois cohomology of an arbitrary relative Galois group $\operatorname{Gal}(E / F)$ of $F$.

\section{1. $\kappa$-Structures}

In this section we define a convenient target category for the generalized Milnor $K$-ring functor. Recall that the Milnor $K$-ring of a field $F$ is a graded ring with degree 1 component $F^{\times}$. Furthermore, the element -1 of $F^{\times}$plays a special role in this ring. The idea is therefore to consider graded rings with a distinguished element in degree 1 , which will play the role of -1 . The resulting category of " $\kappa$ structures" is a slight modification of the " $\kappa$-algebras" defined in [Bass and Tate 1973], in the sense that we require in addition that the degree- 0 component of the graded ring is $\mathbb{Z}$. This turns out to be useful for defining natural constructions in the resulting category, such as extensions (see below) and direct products. Other formal categories that were studied in the context of quadratic form theory (like abstract 
Witt rings, quaternionic structures, or abstract spaces of orderings [Marshall 1980; 1996]) are specializations of the category of $\kappa$-structures in a natural way.

Denote the tensor algebra of an abelian group $\Gamma$ by $\operatorname{Tens}(\Gamma)$. We let

$$
\kappa=\bigoplus_{r=0}^{\infty} \kappa_{r}=\operatorname{Tens}(\{ \pm 1\}),
$$

and denote the nontrivial element of $\kappa_{1} \cong \mathbb{Z} / 2$ by $\varepsilon$. Thus $\kappa_{0}=\mathbb{Z}$ and for all $r \geq 1$, $\kappa_{r}=\left\{0, \varepsilon^{r}\right\} \cong \mathbb{Z} / 2$.

Definition 1.1. A $\kappa$-structure consists of a graded ring $A=\bigoplus_{r=0}^{\infty} A_{r}$ and a graded ring homomorphism $\kappa \rightarrow A$ such that

(i) $A_{0}=\mathbb{Z}$, and the homomorphism $\kappa \rightarrow A$ is the identity in degree 0 ,

(ii) $A_{1}$ generates $A$ as a ring, and

(iii) the image $\varepsilon_{A}$ of $\varepsilon$ in $A$ satisfies $a^{2}=\varepsilon_{A} a=a \varepsilon_{A}$ for all $a \in A_{1}$.

For every $a, b \in A_{1}$ we have $a b+b a=(a+b)^{2}-a^{2}-b^{2}=0$, by (iii). Thus $A$ is anticommutative. A morphism $A \rightarrow B$ of $\kappa$-structures is a graded ring homomorphism which commutes with the structural homomorphisms $\kappa \rightarrow A$ and $\kappa \rightarrow B$.

The category of $\kappa$-structures has direct products. Namely, the direct product $\prod_{i \in I} A_{i}$ of $\kappa$-structures $A_{i}$ for $i \in I$, is defined by $\left(\prod_{i \in I} A_{i}\right)_{0}=\mathbb{Z}$ and $\left(\prod_{i \in I} A_{i}\right)_{r}=$ $\prod_{i \in I}\left(A_{i}\right)_{r}$ for $r \geq 1$, with the natural multiplicative structure. The homomorphism $\kappa_{r} \rightarrow \prod_{i \in I}\left(A_{i}\right)_{r}$ is given by $\varepsilon \mapsto\left(\varepsilon_{A_{i}}\right)_{i \in I}$.

Recall that the tensor product in the category of graded rings is defined by

$$
A \otimes_{\mathbb{Z}} B=\bigoplus_{r=0}^{\infty}\left(\bigoplus_{i+j=r} A_{i} \otimes_{\mathbb{Z}} B_{j}\right),
$$

with the product given by

$$
(a \otimes b)\left(a^{\prime} \otimes b^{\prime}\right)=(-1)^{i^{\prime} j} a a^{\prime} \otimes b b^{\prime}
$$

for $a \in A_{i}, a^{\prime} \in A_{i^{\prime}}, b \in B_{j}, b^{\prime} \in B_{j^{\prime}}$. Given $\kappa$-structures $A, B$, we define their tensor product in the category of $\kappa$-structures to be $A \otimes_{\kappa} B=\left(A \otimes_{\mathbb{Z}} B\right) / I$, where $I$ is the homogeneous ideal generated by $\varepsilon_{A} \otimes 1_{B}-1_{A} \otimes \varepsilon_{B}$. The homomorphism $\kappa \rightarrow A \otimes_{\kappa} B$ is given by $\varepsilon \mapsto \varepsilon_{A} \otimes 1_{B}+I=1_{A} \otimes \varepsilon_{B}+I$. Since $A$ and $B$ are anticommutative, so is $A \otimes_{\mathbb{Z}} B$. Further, given $a \in A_{1}$ and $b \in B_{1}$ we have $\left(a \otimes 1_{B}\right)^{2}=\left(\varepsilon_{A} \otimes 1_{B}\right)\left(a \otimes 1_{B}\right)$ and $\left(1_{A} \otimes b\right)^{2}=\left(1_{A} \otimes \varepsilon_{B}\right)\left(1_{A} \otimes b\right)$, so by the anticommutativity,

$$
\left(a \otimes 1_{B}+1_{A} \otimes b\right)^{2}+I=\left(\varepsilon_{A} \otimes 1_{B}\right)\left(a \otimes 1_{B}+1_{A} \otimes b\right)+I
$$


in $\left(A \otimes_{\kappa} B\right)_{2}$. This implies the first equality in (iii) for $A \otimes_{\kappa} B$. The second is proved similarly, showing that $A \otimes_{\kappa} B$ is a $\kappa$-structure. There are canonical morphisms $\iota: A \rightarrow A \otimes_{\kappa} B$ and $\iota^{\prime}: B \rightarrow A \otimes_{\kappa} B$ with respect to which $A \otimes_{\kappa} B$ is the coproduct of $A$ and $B$ in the category of $\kappa$-structures (in the sense of, e.g., [Lang 1984, I §7]). One has $A \cong A \otimes_{\kappa} \kappa$ and $B \cong \kappa \otimes_{\kappa} B$ via these morphisms.

Next we construct free objects in this category. Let $\Gamma$ be an abelian group. We define $\kappa[\Gamma]$ to be the quotient of $\operatorname{Tens}\left(\kappa_{1} \oplus \Gamma\right)$ by the homogeneous ideal generated by all elements $\varepsilon \otimes \gamma-\gamma \otimes \gamma$, where $\gamma \in \Gamma$. Replacing $\gamma$ by $\varepsilon+\gamma$ one sees that this ideal also contains $\gamma \otimes \varepsilon-\gamma \otimes \gamma$. The obvious embedding $\kappa_{1} \hookrightarrow \kappa_{1} \oplus \Gamma$ induces a graded ring homomorphism $\kappa \rightarrow \kappa[\Gamma]$. Then $\kappa[\Gamma]$ is a $\kappa$-structure satisfying the following universal property (which follows from the universal property of the tensor algebra):

For every $\kappa$-structure $B$ and an abelian group homomorphism $\theta: \Gamma \rightarrow B_{1}$ there exists a unique morphism $\kappa[\Gamma] \rightarrow B$ extending $\theta$.

Given a $\kappa$-structure $A$, we call $A[\Gamma]=A \otimes_{\kappa} \kappa[\Gamma]$ the extension of $A$ by $\Gamma$. When $A=\kappa$ it coincides with our previous notation. This extends Serre's construction mentioned in [Milnor 1970, p. 323]. We identify $(A[\Gamma])_{1}$ with $A_{1} \oplus \Gamma$, and we let $\iota: A \rightarrow A[\Gamma]$ be the canonical morphism.

Lemma 1.2. Let $\varphi: A \rightarrow B$ be a morphism of $\kappa$-structures and let $\theta: \Gamma \rightarrow B_{1}$ be a homomorphism of abelian groups. There exists a unique morphism $A[\Gamma] \rightarrow B$ extending $\theta$ which commutes with $\varphi$ and $\iota$.

Proof. The universal property of $\kappa[\Gamma]$ yields a unique morphism $\kappa[\Gamma] \rightarrow B$ extending $\theta$. Now use the fact that the tensor product is a coproduct.

Corollary 1.3. For a $\kappa$-structure $A$ and abelian groups $\Gamma_{1}, \Gamma_{2}$ one has

$$
\left(A\left[\Gamma_{1}\right]\right)\left[\Gamma_{2}\right] \cong A\left[\Gamma_{1} \oplus \Gamma_{2}\right] .
$$

Example 1.4. Let $A$ be a $\kappa$-structure and let $\Gamma$ be a cyclic group with generator $\gamma$. For every $i \geq 1$, we have $\gamma^{i}=\varepsilon_{A}^{i-1} \gamma$ in $A[\Gamma]$, by (iii) of Definition 1.1. It follows that $(A[\Gamma])_{r}=A_{r} \oplus\left(A_{r-1} \otimes_{\mathbb{Z}} \Gamma\right)$ for $r \geq 1$.

\section{The functor $K_{*}^{M}(F) / S$}

Let $F$ be a field and let $S$ be a subgroup of $F^{\times}$. For $r \geq 0$, let

$$
\left(F^{\times} / S\right)^{\otimes r}=\left(F^{\times} / S\right) \otimes_{\mathbb{Z}} \cdots \otimes_{\mathbb{Z}}\left(F^{\times} / S\right) \quad(r \text { times }) .
$$

Let $\operatorname{St}_{F, r}(S)$ be the subgroup of $\left(F^{\times} / S\right)^{\otimes r}$ generated by all elements $a_{1} S \otimes \cdots \otimes a_{r} S$ such that $1 \in a_{i} S+a_{j} S$ for some $i \neq j$. Generalizing standard terminology, we call such elements Steinberg elements. Let

$$
K_{r}^{M}(F) / S=\left(F^{\times} / S\right)^{\otimes r} / \operatorname{St}_{F, r}(S) .
$$


In particular, $K_{0}^{M}(F) / S=\mathbb{Z}$ and $K_{1}^{M}(F) / S=F^{\times} / S$. For $t \geq 0$, one has

$$
\operatorname{St}_{F, r}(S) \otimes_{\mathbb{Z}}\left(F^{\times} / S\right)^{\otimes t} \subseteq \operatorname{St}_{F, r+t}(S), \quad\left(F^{\times} / S\right)^{\otimes t} \otimes_{\mathbb{Z}} \operatorname{St}_{F, r}(S) \subseteq \operatorname{St}_{F, r+t}(S) .
$$

Therefore

$$
K_{*}^{M}(F) / S=\bigoplus_{r=0}^{\infty} K_{r}^{M}(F) / S
$$

is a graded ring respect to the multiplication induced by the tensor product. We call it the Milnor $K$-ring of $F$ modulo $S$. Given $a_{1}, \ldots, a_{r} \in F^{\times}$, we denote the image of $a_{1} S \otimes \cdots \otimes a_{r} S$ in $K_{r}^{M}(F) / S$ by $\left\{a_{1}, \ldots, a_{r}\right\}_{S}$.

When $S=\{1\}$ we obtain the classical Milnor $K$-ring $K_{*}^{M}(F)=\bigoplus_{r=0}^{\infty} K_{r}^{M}(F)$ of $F$ as in [Milnor 1970]. In this case we write as usual $\left\{a_{1}, \ldots, a_{n}\right\}$ for $\left\{a_{1}, \ldots, a_{n}\right\}_{S}$. In general, we have graded ring homomorphisms $\operatorname{Tens}\left(F^{\times} / S\right) \rightarrow K_{*}^{M}(F) / S$ and $K_{*}^{M}(F) \rightarrow K_{*}^{M}(F) / S$.

Define a graded ring homomorphism $\kappa \rightarrow K_{*}^{M}(F) / S$ by setting $\varepsilon \mapsto-S \in F^{\times} / S$. Since the identities $\{a, a\}_{S}=\{-1, a\}_{S}=\{a,-1\}_{S}$ of part (iii) of Definition 1.1 are well known to hold when $S=\{1\}$ [Milnor 1970, §1], they also hold in $K_{*}^{M}(F) / S$. Hence $K_{*}^{M}(F) / S$ is a $\kappa$-structure.

Proposition 2.1. For positive integers $m, r$ and for $S=\left(F^{\times}\right)^{m}$ we have

$$
K_{r}^{M}(F) / S=K_{r}^{M}(F) / m \text {. }
$$

Proof. There is an obvious graded ring homomorphism $\varphi: K_{r}^{M}(F) / m \rightarrow K_{r}^{M}(F) / S$ commuting with the canonical projections from $\left(F^{\times}\right)^{\otimes r}$. Conversely, suppose $a_{1}, \ldots, a_{r} \in F^{\times}$and $a_{1} S \otimes \cdots \otimes a_{r} S \in \mathrm{St}_{F, r}(S)$, that is, $1=a_{i} \alpha^{m}+a_{j} \beta^{m}$ for some $i<j$ and $\alpha, \beta \in F^{\times}$. Then

$$
\left\{a_{1}, \ldots, a_{r}\right\} \in\left\{a_{1}, \ldots, a_{i} \alpha^{m}, \ldots, a_{j} \beta^{m}, \ldots, a_{r}\right\}+m K_{r}^{M}(F)=m K_{r}^{M}(F) .
$$

We obtain a projection $\psi: K_{r}^{M}(F) / S \rightarrow K_{r}^{M}(F) / m$ which also commutes with the projections from $\left(F^{\times}\right)^{\otimes r}$. Thus $\varphi$ and $\psi$ are inverse maps, hence isomorphisms.

We consider the class of all pairs $(F, S)$ where $F$ is a field and $S \leq F^{\times}$as a category, in which morphisms $(F, S) \rightarrow\left(F_{1}, S_{1}\right)$ are pairs of compatible embeddings $F \hookrightarrow F_{1}, S \hookrightarrow S_{1}$. For such a pair and for $r \geq 0$, we have a group homomorphism $\left(F^{\times} / S\right)^{\otimes r} \rightarrow\left(F_{1}^{\times} / S_{1}\right)^{\otimes r}$ mapping $\operatorname{St}_{F, r}(S)$ to $\operatorname{St}_{F_{1}, r}\left(S_{1}\right)$. It therefore induces a $\kappa$ structure morphism Res : $K_{*}^{M}(F) / S \rightarrow K_{*}^{M}\left(F_{1}\right) / S_{1}$, which we call the restriction morphism. The map $(F, S) \mapsto K_{*}^{M}(F) / S$ is thus a covariant functor from the category of pairs $(F, S)$ to the category of $\kappa$-structures.

A topology on a field $F$ is called a ring topology if the addition and multiplication maps $F \times F \rightarrow F$ are continuous. We will need: 
Proposition 2.2. Let $\mathcal{T}$ be a ring topology on a field $F_{1}$ and let $F$ be a subfield of $F_{1}$ which is $\mathscr{T}$-dense in $F_{1}$. Let $S$ be a subgroup of $F^{\times}$and let $S_{1}$ be a $\mathcal{T}$ open subgroup of $F_{1}^{\times}$containing $S$. Then $\operatorname{Res}: K_{*}^{M}(F) / S \rightarrow K_{*}^{M}\left(F_{1}\right) / S_{1}$ is an epimorphism. When $S=F \cap S_{1}$, it is an isomorphism.

Proof. For every $a \in F_{1}^{\times}$, we have $F \cap a S_{1} \neq \varnothing$ by the density assumption. Hence the natural homomorphism $F^{\times} / S \rightarrow F_{1}^{\times} / S_{1}$ is surjective. Consequently, so is Res : $K_{*}^{M}(F) / S \rightarrow K_{*}^{M}\left(F_{1}\right) / S_{1}$.

Suppose that $S=F \cap S_{1}$. For each $r$ the induced map $\left(F^{\times} / S\right)^{\otimes r} \rightarrow\left(F_{1}^{\times} / S_{1}\right)^{\otimes r}$ is an isomorphism. Therefore the injectivity of Res would follow by a snake lemma argument once we show that the induced map $\operatorname{St}_{F, r}(S) \rightarrow \mathrm{St}_{F_{1}, r}\left(S_{1}\right)$ is surjective. To this end, take a generator $a_{1} S_{1} \otimes \cdots \otimes a_{r} S_{1} \in \operatorname{St}_{F_{1}, r}\left(S_{1}\right)$, where $a_{1}, \ldots, a_{r} \in F_{1}^{\times}$ and $1 \in a_{i} S_{1}+a_{j} S_{1}$ for some distinct $i, j$. By continuity, there exist nonempty $\mathscr{T}_{\text {- }}$ open subsets $V, W \subseteq S_{1}$ such that $a_{i} V+a_{j} W \subseteq S_{1}$. Using the density assumption we find $x_{1}, \ldots, x_{r} \in F$ with $x_{i} \in a_{i} V, x_{j} \in a_{j} W$, and $x_{l} \in a_{l} S_{1}$ for all $l \neq i, j$. Then $x_{i}+x_{j} \in S_{1} \cap F=S$, so $x_{1} S \otimes \cdots \otimes x_{r} S \in \operatorname{St}_{F, r}(S)$. Furthermore, $x_{1} S \otimes \cdots \otimes x_{r} S$ maps to $a_{1} S_{1} \otimes \cdots \otimes a_{r} S_{1}$ under the homomorphism above, as required.

\section{Orderings}

Let again $F$ be a field, and let $S$ be a subgroup of $F^{\times}$. Following standard terminology (see, e.g., [Neukirch et al. 2000, p. 191]), we call the map $\operatorname{Bock}_{F, S}: F^{\times} / S \rightarrow$ $K_{2}^{M}(F) / S,\{x\}_{S} \mapsto\{x\}_{S}^{2}=\{x,-1\}_{S}$, the Bockstein operator of the subgroup $S$ of $F$. It is clearly a group homomorphism.

Lemma 3.1. If Bock $_{F, S}$ is injective then $S$ is additively closed.

Proof. It suffices to show that $1+S \subseteq S$. To this end take $s \in S$. Then

$$
\operatorname{Bock}_{F, S}\left(\{1+s\}_{S}\right)=\{1+s,-1\}_{S}=\{1+s,-s\}_{S}=0 .
$$

By injectivity, $\{1+s\}_{S}=0$, so $1+s \in S$.

By an ordering on $F$, we mean an additively closed subgroup $P$ of $F^{\times}$such that $F^{\times}=P \cup-P$. Recall that a ring is reduced if it has no nonzero nilpotent elements. The following fact is a variant of [Bass and Tate 1973, I, Theorem (3.1)].

Proposition 3.2. The following conditions are equivalent:

(a) $K_{*}^{M}(F) / S \cong \kappa$ as $\kappa$-structures;

(b) $F^{\times}=S \bullet-S$ and $K_{*}^{M}(F) / S$ is reduced;

(c) $F^{\times}=S \cup-S$ and $\{-1,-1\}_{S} \neq 0$;

(d) $S$ is an ordering on $F$. 
Proof. (a) $\Rightarrow$ (b) $\Rightarrow$ (c): Immediate.

(c) $\Rightarrow(\mathrm{d})$ : By Lemma 3.1 and the assumptions, $S$ is additively closed. The rest is clear.

(d) $\Rightarrow$ (a): We first show that $\operatorname{St}_{F, r}(S)$ is trivial for all $r \geq 2$. Take $a_{1}, \ldots, a_{r} \in F^{\times}$ with $1 \in a_{i} S+a_{j} S$ for some distinct $1 \leq i, j \leq r$. If $a_{i}, a_{j}$ were both in $-S$ then we would get $-1 \in S+S \subseteq S$, a contradiction. Hence at least one of $a_{i}, a_{j}$ must be in $S$. It follows that $a_{1} S \otimes \cdots \otimes a_{r} S=1$ in $\left(F^{\times} / S\right)^{\otimes r}$, as claimed.

Consequently, $K_{*}^{M}(F) / S=\operatorname{Tens}\left(F^{\times} / S\right) \cong \operatorname{Tens}(\{ \pm 1\})=\kappa$ as graded rings. Further, this is a $\kappa$-structure isomorphism.

A preordering on $F$ is an additively closed subgroup $S$ of $F^{\times}$containing $\left(F^{\times}\right)^{2}$ but not -1 . Preorderings can be characterized $K$-theoretically as follows.

Proposition 3.3. Suppose that $\left(F^{\times}\right)^{2} \leq S<F^{\times}$. The following conditions are equivalent:

(a) $S$ is a preordering on $F$;

(b) Bock $_{F, S}$ is injective.

Proof. (a) $\Rightarrow$ (b): Let $x \in F^{\times}$satisfy $\{x\}_{S}^{2}=0$ and let $P$ be an ordering on $F$ containing $S$. Then $\{x\}_{P}^{2}=0$, and since $K_{*}^{M}(F) / P \cong \kappa$ is reduced (Proposition 3.2), $\{x\}_{P}=0$, i.e., $x \in P$. As a preordering, $S$ is the intersection of all the orderings $P$ containing it [Lam 1983, Theorem 1.6]. Consequently, $x \in S$, as desired.

(b) $\Rightarrow$ (a): In light of Lemma 3.1, $S$ is additively closed. By assumption, there exists $x \in F^{\times} \backslash S$. By injectivity, $\{x,-1\}_{S} \neq 0$. Hence $-1 \notin S$, so $S$ is a preordering.

\section{The cyclic case}

Using the $K$-theoretic analysis of orderings obtained in the previous section, we can now completely describe $K_{*}^{M}(F) / S$ when $F^{\times} / S$ is a finite cyclic group.

Theorem 4.1. Let $F$ be a field and let $S$ be a subgroup of $F^{\times}$such that $F^{\times} / S$ is finite and cyclic. Then one of following holds:

(a) $K_{r}^{M}(F) / S=0$ for all $r \geq 2$;

(b) $\left(F^{\times}: S\right)=2 m$ with $m$ odd, and there exists a unique ordering $P$ on $F$ containing $S$. Furthermore, $\operatorname{Res}: K_{*}^{M}(F) / S \rightarrow K_{*}^{M}(F) / P(\cong \kappa)$ is an isomorphism in all degrees $r \geq 2$.

Proof. Let $p_{1}^{d_{1}} \cdots p_{n}^{d_{n}}$ be the primary decomposition of $\left(F^{\times}: S\right)$. For each $1 \leq$ $i \leq n$, choose $a_{i} \in F^{\times}$such that the coset $\left\{a_{i}\right\}_{S}$ generates the $p_{i}$-primary part of $F^{\times} / S$. Let $a=a_{1} \cdots a_{n}$. Then the coset $\{a\}_{S}$ generates $F^{\times} / S$, and one has $\{a, a\}_{S}=\{a,-1\}_{S}=\sum_{i=1}^{n}\left\{a_{i},-1\right\}_{S}$. 
Assume that (a) does not hold, i.e., $K_{r}^{M}(F) / S \neq 0$ for some $r \geq 2$. Since the canonical map $\left(F^{\times} / S\right)^{r} \rightarrow K_{r}^{M}(F) / S$ is multilinear, $\{a, \ldots, a\}_{S}$ generates $K_{r}^{M}(F) / S$. Hence $\{a, \ldots, a\}_{S} \neq 0$, and therefore $\{a, a\}_{S} \neq 0$. It follows that $\left\{a_{i},-1\right\}_{S} \neq 0$ for some $1 \leq i \leq n$. We obtain that the orders of $\left\{a_{i},-1\right\}_{S}$ and of $\{-1\}_{S}$ are precisely 2 . Furthermore, $p_{i}^{d_{i}}\left\{a_{i},-1\right\}_{S}=0$, so we must have $p_{i}=2$. Therefore $2^{d_{i}-1}\left\{a_{i}\right\}_{S}=\{-1\}_{S}$, and we get

$$
2^{d_{i}-1}\left\{a_{i},-1\right\}_{S}=2^{d_{i}-1}\left\{a_{i}, a_{i}\right\}_{S}=\left\{a_{i},-1\right\}_{S} \neq 0 .
$$

This implies that $d_{i}=1$. Consequently, $\left(F^{\times}: S\right)=2 m$, with $m$ odd.

Let $P$ be the unique subgroup of $F^{\times}$of index 2 which contains $S$. Then $P / S$ is cyclic of order $m$, and is generated by $\left\{a^{2}\right\}_{S}$. Since $\{-1\}_{S}$ has order 2 in $F^{\times} / S$, it is not in $P / S$. Therefore $F^{\times}=P \cup-P$.

Next we claim that $1+P \subseteq P$. Indeed, suppose that $x \in P$. In particular, $x \neq-1$. Take $s, t$ with $-x \in a^{s} S$ and $1+x \in a^{t} S$. Then

$$
0=\{-x, 1+x\}_{S}=\left\{a^{s}, a^{t}\right\}_{S}=s t\{a, a\}_{S} .
$$

Now $-x \notin P$, so $s$ is odd. But $\{a, a\}_{S}=\{a,-1\}_{S}$ has order 2. It follows that $t$ must be even, i.e., $1+x \in P$. Therefore $P$ is additively closed, hence an ordering.

Finally, for every $r$, the functorial map $K_{r}^{M}(F) / S \rightarrow K_{r}^{M}(F) / P$ is clearly surjective. When $r \geq 2$, the group $K_{r}^{M}(F) / S$ is generated by $\{a, a, \ldots, a\}_{S}=$ $\{a,-1, \ldots,-1\}_{S}$, so it has order at most 2. By Proposition 3.2, $K_{r}^{M}(F) / P$ has order 2. Consequently, the above map is an isomorphism, and (b) holds.

For the uniqueness part of (b), assume that $S \leq P^{\prime}<F^{\times}$is another ordering on $F$. Then $4\left|\left(F^{\times}: P \cap P^{\prime}\right)\right|\left(F^{\times}: S\right)=2 m$, contrary to the fact that $m$ is odd.

Corollary 4.2. Let $S$ be a subgroup of $F^{\times}$with $F^{\times} / S$ cyclic of prime power order. Then either $K_{r}^{M}(F) / S=0$ for all $r \geq 2$, or $S$ is an ordering (hence $\left.K_{*}^{M}(F) / S \cong \kappa\right)$.

As mentioned in the introduction, Theorem 4.1 generalizes the well-known fact that $K_{2}^{M}(F)=0$ for a finite field $F$ [Milnor 1970, Example 1.5; Fesenko and Vostokov 1993, IX, Proposition 1.3]. Indeed, $F^{\times}$is cyclic [Lang 1984, VII §5, Theorem 11] and since char $F>0$, there are no orderings on $F$.

\section{5. $S$-compatible valuations}

Recall that a (Krull) valuation on a field $F$ is a group homomorphism $v$ from $F^{\times}$ into an ordered abelian group $(\Gamma, \leq)$ such that $v(x+y) \geq \min \{v(x), v(y)\}$ for all $x, y \in F$ with $x \neq-y$. One defines $v(0)$ to be a formal value $+\infty$ which is strictly larger than every value in $\Gamma$. Let $O_{v}$ be the valuation ring of $v$, and $\mathfrak{m}_{v}$ its maximal ideal. Thus $x \in F$ lies in $O_{v}$ (respectively $\mathfrak{m}_{v}$ ) if and only if $v(x) \geq 0$ (respectively $v(x)>0$ ). Let $O_{v}^{\times}$be the unit group of $O_{v}$, let $G_{v}=1+\mathfrak{m}_{v}$ be the 
group of principal units of $v$, let $\bar{F}_{v}=O_{v} / \mathfrak{m}_{v}$ be the residue field of $v$, and let $\pi_{v}: O_{v} \rightarrow \bar{F}_{v}, a \mapsto \bar{a}$, be the canonical projection.

Let $S$ a subgroup of $F^{\times}$. Its push-down $\bar{S}_{v}=\pi_{v}\left(S \cap O_{v}^{\times}\right)$under $v$ is a subgroup of $\bar{F}_{v}^{\times}$. The maps $v$ and $\pi_{v}$ induce short exact sequences of abelian groups

$$
1 \rightarrow S \cap O_{v}^{\times} \rightarrow S \stackrel{v}{\longrightarrow} v(S) \rightarrow 0
$$

and

$$
1 \rightarrow S \cap G_{v} \rightarrow S \cap O_{v}^{\times} \stackrel{\pi_{v}}{\longrightarrow} \bar{S}_{v} \rightarrow 1 .
$$

In particular, this holds for $S=F^{\times}$. The snake lemma therefore gives rise to canonical exact sequences

$$
1 \rightarrow O_{v}^{\times} /\left(S \cap O_{v}^{\times}\right) \rightarrow F^{\times} / S \stackrel{v^{*}}{\longrightarrow} v\left(F^{\times}\right) / v(S) \rightarrow 0
$$

and

$$
1 \rightarrow G_{v} /\left(S \cap G_{v}\right) \rightarrow O_{v}^{\times} /\left(S \cap O_{v}^{\times}\right) \stackrel{\pi_{v}^{*}}{\longrightarrow} \bar{F}_{v}^{\times} / \bar{S}_{v} \rightarrow 1 .
$$

Following [Arason et al. 1987], we say that the valuation $v$ is $S$-compatible if $G_{v} \leq S$. (When $S=\left(F^{\times}\right)^{p}$ for $p$ prime and char $\bar{F}_{v} \neq p$, this is a weak form of Hensel's lemma; see [Wadsworth 1983, Proposition 1.2].) Then the sequences (5-1) and (5-2) combine to a single canonical short exact sequence

$$
1 \rightarrow \bar{F}_{v}^{\times} / \bar{S}_{v} \stackrel{\eta}{\longrightarrow} F^{\times} / S \stackrel{v^{*}}{\longrightarrow} v\left(F^{\times}\right) / v(S) \rightarrow 0,
$$

where for $a \in O_{v}^{\times}$with residue $\bar{a}$ we set $\eta\left(\{\bar{a}\}_{\bar{S}_{v}}\right)=\{a\}_{S}$.

We will be interested in situations where (5-1) splits. For example, this is so in the following cases:

(1) $v\left(F^{\times}\right) \cong \mathbb{Z}$ and $S=\{1\}$. Then a section of $v^{*}$ corresponds to a choice of a uniformizer for $v$.

(2) $\left(F^{\times}\right)^{p} \leq S$ for some prime number $p$. In fact, then $F^{\times} / S$ and $v\left(F^{\times}\right) / v(S)$ are free $(\mathbb{Z} / p)$-modules.

(3) $\left(F^{\times}\right)^{q} \leq S \leq\left(F^{\times}\right)^{q} O_{v}^{\times}$, where $q=p^{s}$ is a prime power. Indeed, the group $v\left(F^{\times}\right)$is torsion-free, hence a flat $\mathbb{Z}$-module. Thus $v\left(F^{\times}\right) / v(S)=v\left(F^{\times}\right) / q$ is a flat $\mathbb{Z} / q$-module. Since $\mathbb{Z} / q$ is a nilpotent local ring, it is a consequence of the Nakayama lemma [Matsumura 1980, 3.G] that $v\left(F^{\times}\right) / q$ is a free $\mathbb{Z} / q$ module.

We now obtain a connection between valuations and extensions of $\kappa$-structures, in the sense of Section 1.

Theorem 5.1. Let $F$ be a field and let $S$ be a subgroup of $F^{\times}$. Every section of $(5-1)$ canonically induces an epimorphism of $\kappa$-structures

$$
K_{*}^{M}(F) / S \longrightarrow\left(K_{*}^{M}\left(\bar{F}_{v}\right) / \bar{S}_{v}\right)\left[v\left(F^{\times}\right) / v(S)\right] .
$$


This morphism is injective if and only if $v$ is $S$-compatible.

Proof. Let $\theta: v\left(F^{\times}\right) / v(S) \rightarrow F^{\times} / S$ be a section of $v^{*}$. Take $S \leq \Delta \leq F^{\times}$with $\Delta / S=\operatorname{Im}(\theta)$. Then $F^{\times} / S=\left(S O_{v}^{\times} / S\right) \times(\Delta / S)$. Thus every $x \in F^{\times}$can be written as $x=a b$ with $a \in O_{v}^{\times}$and $b \in \Delta$. We set $\bar{a}=\pi_{v}(a)$ and write $[v(b)]_{S}$ for the coset of $v(b)$ in $v\left(F^{\times}\right) / v(S)$. We obtain a well-defined group epimorphism

$$
F^{\times} / S \rightarrow\left(\bar{F}_{v}^{\times} / \bar{S}_{v}\right) \oplus\left(v\left(F^{\times}\right) / v(S)\right), \quad\{x\}_{S} \mapsto\{\bar{a}\}_{\bar{S}_{v}}+[v(b)]_{S} .
$$

This abelian group epimorphism uniquely extends to a graded ring epimorphism

$$
\lambda: \operatorname{Tens}\left(F^{\times} / S\right) \rightarrow\left(K_{*}^{M}\left(\bar{F}_{v}\right) / \bar{S}_{v}\right)\left[v\left(F^{\times}\right) / v(S)\right] .
$$

We claim that $\lambda$ is trivial on $\mathrm{St}_{F, r}(S)$ for all $r$. It suffices to show that when $x, y \in F^{\times}$and $1 \in x S+y S$, we have $\lambda\left(\{x\}_{S} \otimes\{y\}_{S}\right)=0$. We may assume that $1=x+y$. Write $x=a b$ and $y=c d$, with $a, c \in O_{v}^{\times}$and $b, d \in \Delta$. Then

$$
\begin{aligned}
\lambda(x S \otimes y S) & =\left(\{\bar{a}\}_{\bar{S}_{v}}+[v(b)]_{S}\right) \cdot\left(\{\bar{c}\}_{\bar{S}_{v}}+[v(d)]_{S}\right) \\
& =\{\bar{a}, \bar{c}\}_{\bar{S}_{v}}+\left(\{\bar{a}\}_{\bar{S}_{v}} \cdot[v(d)]_{S}-\{\bar{c}\}_{\bar{S}_{v}} \cdot[v(b)]_{S}\right)+[v(b)]_{S} \cdot[v(d)]_{S} .
\end{aligned}
$$

To show that this expression vanishes, we distinguish between four cases:

Case I: $x \in G_{v}$. Here we can take $a=x$ and $b=1$. Then $\{\bar{a}\}_{\bar{S}_{v}}=0$ and $[v(b)]_{S}=0$, so the assertion is clear.

Case II: $x \in \mathfrak{m}_{v}$. Then $y \in G_{v}$, so we can take $c=y$ and $d=1$. Hence $\{\bar{c}\}_{\bar{S}_{v}}=0$ and $[v(d)]_{S}=0$, and we are done again.

Case III: $x \in O_{v}^{\times} \backslash G_{v}$. Then $y=1-x \in O_{v}^{\times}$, so we can take $a=x, b=1, c=y$, and $d=1$. Hence $\lambda(x S \otimes y S)=\{\bar{x}, \overline{1-x}\}_{\bar{S}_{v}}=0$ once again.

Case IV: $x^{-1} \in \mathfrak{m}_{v}$. For any $a, b$ as above, $y=a\left(x^{-1}-1\right) \cdot b$, with $a\left(x^{-1}-1\right) \in O_{v}^{\times}$. Thus we may take $c=a\left(x^{-1}-1\right)$ and $d=b$. Then $\{\bar{c}\}_{\bar{S}_{v}}=\{-\bar{a}\}_{\bar{S}_{v}}$. Further, $\{\bar{a}\}_{\bar{S}_{v}}-\{-\bar{a}\}_{\bar{S}_{v}}=\{-\overline{1}\}_{\bar{S}_{v}}$ and $\{\bar{a},-\bar{a}\}_{\bar{S}_{v}}=0$. It follows that

$$
\lambda(x S \otimes y S)=\{-\overline{1}\}_{\bar{S}_{v}} \cdot[v(b)]_{S}+[v(b)]_{S} \cdot[v(b)]_{S}=0,
$$

using property (iii) of Definition 1.1.

This proves the claim. Consequently, $\lambda$ induces an epimorphism of $\kappa$-structures

$$
\bar{\lambda}: K_{*}^{M}(F) / S \longrightarrow\left(K_{*}^{M}\left(\bar{F}_{v}\right) / \bar{S}_{v}\right)\left[v\left(F^{\times}\right) / v(S)\right],
$$

as desired.

For the second assertion of the theorem, suppose that $v$ is $S$-compatible. Then (5-3) is exact. The abelian group monomorphism $\eta$ of (5-3) induces a morphism Tens $\left(\bar{F}_{v}^{\times} / \bar{S}_{v}\right) \rightarrow \operatorname{Tens}\left(F^{\times} / S\right)$ of graded rings. Since $G_{v} \leq S$, it maps $\mathrm{St}_{\bar{F}_{v}, r}\left(\bar{S}_{v}\right)$ into $\operatorname{St}_{F, r}(S)$ for every $r \geq 1$. Hence it induces a $\kappa$-structure morphism 
$K_{*}^{M}\left(\bar{F}_{v}\right) / \bar{S}_{v} \rightarrow K_{*}^{M}(F) / S$. By the universal property of extensions (Lemma 1.2), there exists a unique $\kappa$-structure morphism $\bar{v}$ which extends the section $\theta$ and for which the following diagram commutes (where $\iota$ is the canonical morphism as in Section 1):

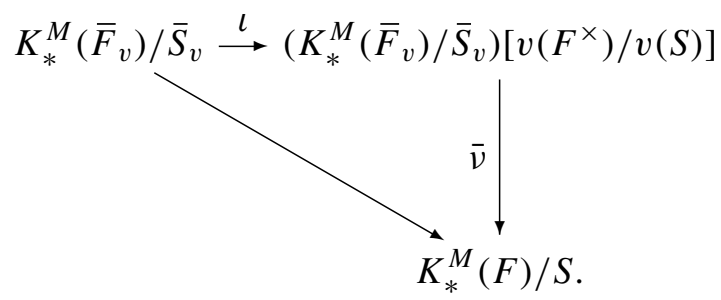

In degree $1, \bar{v}$ coincides with the isomorphism $\eta \oplus \theta$. Hence it is surjective in all degrees. By construction, $\bar{\lambda}$ is given in degree 1 by the map (5-4). It follows that $\bar{\lambda} \circ \bar{v}=$ id in degree 1 , and therefore in all degrees. This proves that $\bar{v}$ is injective. Therefore both $\bar{v}$ and $\bar{\lambda}$ are isomorphisms.

Conversely, suppose that $\bar{\lambda}$ is an isomorphism. Its definition in degree 1 shows that it maps $G_{v} S / S$ trivially. Hence $G_{v} \leq S$, as required.

Remark 5.2. When $v$ is a discrete valuation and $S=\{1\}$, the first part of Theorem 5.1 is due to Bass and Tate [1973, I, Proposition 4.3]. They also prove its second part when $(F, v)$ is a complete, discretely valued field with positive residue characteristic prime to $m$ and when $S=\left(F^{\times}\right)^{m}$ [Bass and Tate 1973, I, Corollary 4.7]. Note that in the latter case, $v$ is $S$-compatible by Hensel's lemma. Wadsworth $[1983, \S 2]$ proves Theorem 5.1 for any valued field $(F, v)$ when $S=\left(F^{\times}\right)^{q} G_{v}$ and $q$ is a prime power.

Remark 5.3. The epimorphism of Theorem 5.1 is functorial in the following sense: suppose $\left(F_{1}, v_{1}\right)$ is a valued field extension of $(F, v)$, and suppose that $S \leq F^{\times}$, $S_{1} \leq F_{1}^{\times}$, and $S \leq S_{1}$. Further assume there exist homomorphic sections $\theta$ and $\theta_{1}$ of the projections

$$
v^{*}: F^{\times} / S \rightarrow v\left(F^{\times}\right) / v(S), \quad v_{1}^{*}: F_{1}^{\times} / S_{1} \rightarrow v_{1}\left(F_{1}^{\times}\right) / v_{1}\left(S_{1}\right)
$$

induced by $v$ and $v_{1}$, respectively. Moreover, suppose that the following square commutes:

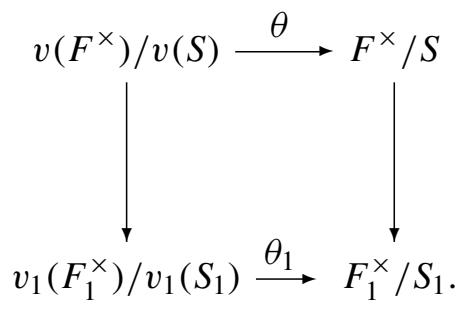


Then the epimorphisms given in Theorem 5.1 and the restriction morphisms induce a square:

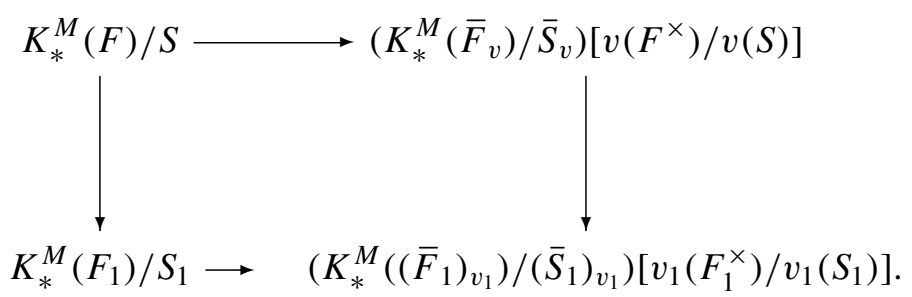

This square commutes in degree 1 , hence in all degrees.

Remark 5.4. There are partial converses to Theorem 5.1. Namely, if $S=\left(F^{\times}\right)^{p}$ for a prime number $p$ and if $K_{*}^{M}(F) / S$ is an extension of some $\kappa$-structure by $(\mathbb{Z} / p)^{d}$, then apart from some well-understood exceptional cases, $F$ is equipped with an $S$-compatible valuation $v$ with $v\left(F^{\times}\right) / p v\left(F^{\times}\right) \cong(\mathbb{Z} / p)^{d}$. Indeed, this follows from the results of [Jacob 1981; Ware 1981; Arason et al. 1987; Hwang and Jacob 1995]; see [Efrat 1999] for a $K$-theoretic formulation of this line of results.

\section{A vanishing theorem}

Recall that a valuation $v$ on $F$ induces a ring topology $\mathscr{T}_{v}$ on $F$, with basis consisting of all sets $a+b O_{v}$, where $a, b \in F$ and $b \neq 0$. For $0<\gamma \in v\left(F^{\times}\right)$the set

$$
W_{\gamma}=\left\{x \in F^{\times} \mid v(1-x) \geq \gamma\right\}
$$

is a $\mathscr{T}_{v}$-open subgroup of $G_{v}=1+\mathfrak{m}_{v}$.

Lemma 6.1. Let $v$ be a valuation on the field $F$. Let $S$ be a subgroup of $F^{\times}$such that $G_{v} /\left(S \cap G_{v}\right)$ is a finitely generated group. Then there exists $0<\gamma \in v\left(F^{\times}\right)$ such that

(i) $S G_{v}=S W_{\gamma}$, and

(ii) if char $\bar{F}_{v}=p$ then $1+p O_{v} \leq W_{\gamma}$.

Proof. We choose $a_{1}, \ldots, a_{n} \in \mathfrak{m}_{v}$ such that the cosets of $1-a_{i}, i=1, \ldots, n$, generate $G_{v} /\left(S \cap G_{v}\right)$. Hence $\left(1-a_{i}\right) S, i=1, \ldots, n$, generate $S G_{v} / S$. Take any $0<\gamma \leq \min \left\{v\left(a_{1}\right), \ldots, v\left(a_{n}\right)\right\}$. Then $1-a_{i} \in W_{\gamma}, i=1, \ldots, n$. Combined with $W_{\gamma} \leq G_{v}$, this shows that $S W_{\gamma} / S=S G_{v} / S$. When char $\bar{F}_{v}=p$ we take

$$
\gamma=\min \left\{v(p), v\left(a_{1}\right), \ldots, v\left(a_{n}\right)\right\} .
$$

One says that the valuation $v$ on $F$ has rank 1 (or that it is Archimedean) if $v\left(F^{\times}\right)$embeds in $\mathbb{R}$ as an ordered abelian group. Equivalently, for every $0<$ 
$\alpha, \gamma \in v\left(F^{\times}\right)$there exists a positive integer $s$ such that $\alpha<s \gamma$ [Bourbaki 1972, VI §4.5, Proposition 8].

Theorem 6.2. Let $v$ be a valuation of rank 1 on the field $F$. Let $S$ be a $\mathscr{T}_{v^{-}}$ open subgroup of $F^{\times}$such that $F^{\times} / S$ is finitely generated and $F^{\times}=S G_{v}$. Then $K_{r}^{M}(F) / S=0$ for all $r \geq 2$.

Proof. It suffices to show that $a S \otimes b S \in \mathrm{St}_{F, 2}(S)$ for $a, b \in G_{v}$. Suppose that this is not the case. In particular, $a, b \notin S$. Lemma 6.1 yields $0<\gamma \in v\left(F^{\times}\right)$such that $F^{\times}=S G_{v}=S W_{\gamma}$.

We define inductively a sequence $c_{1}, c_{2}, \ldots \in G_{v}$ such that for each $i$,

$$
1-c_{i} \in(1-b)\left(1-W_{\gamma}\right)^{i-1}, \quad a S \otimes b c_{i}^{-1} S \in \operatorname{St}_{F, 2}(S) .
$$

We can take $c_{1}=b$. Next suppose that $c_{i}$ has already been constructed. Since $a S \otimes b S \notin \operatorname{St}_{F, 2}(S)$ we have $c_{i} \neq 1$. Choose $y_{i} \in S$ such that $a /\left(1-c_{i}^{-1}\right) \in y_{i} W_{\gamma}$. As $a \notin S$ and $y_{i} \in S$, we may define $c_{i+1}=c_{i}\left(1-y_{i}^{-1} a\right)$. Since $c_{i} \in G_{v}$ we have $y_{i}^{-1} a \in\left(1-c_{i}^{-1}\right) W_{\gamma} \subseteq \mathfrak{m}_{v}$. Hence $c_{i+1} \in G_{v}$. Now

$$
\frac{1-c_{i+1}}{1-c_{i}}=1-\frac{y_{i}^{-1} a}{1-c_{i}^{-1}} \in 1-W_{\gamma},
$$

so by the induction hypothesis, $1-c_{i+1} \in(1-b)\left(1-W_{\gamma}\right)^{i}$. Furthermore,

$$
\begin{aligned}
a S \otimes b c_{i+1}^{-1} S & =a S \otimes b c_{i}^{-1} S-a S \otimes\left(1-y_{i}^{-1} a\right) S \\
& =a S \otimes b c_{i}^{-1} S-y_{i}^{-1} a S \otimes\left(1-y_{i}^{-1} a\right) S \in \operatorname{St}_{F, 2}(S) .
\end{aligned}
$$

This completes the inductive construction.

Since $v$ has rank 1 , the sets $\left(1-W_{\gamma}\right)^{s}, s=1,2,3, \ldots$, form a local basis for $\mathscr{T}_{v}$ at 0 . As $b \neq 1$, the set $(1-b)^{-1}(1-S)$ is a $\mathscr{T}_{v}$-open neighborhood of 0 . Hence there exists a positive integer $t$ such that $\left(1-W_{\gamma}\right)^{t} \subseteq(1-b)^{-1}(1-S)$. Then $1-c_{t+1} \in(1-b)\left(1-W_{\gamma}\right)^{t} \subseteq 1-S$, and so $c_{t+1} \in S$. We conclude that $a S \otimes b S=a S \otimes b c_{t+1}^{-1} S \in \operatorname{St}_{F, 2}(S)$, a contradiction.

\section{Wild valuations of rank 1}

In this section we study $K_{*}^{M}(F)$ when $F$ is a field of characteristic 0 equipped with a valuation $v$ such that char $\bar{F}_{v}=p>0$. First we assume that $v$ is a discrete valuation. Thus $\mathfrak{m}_{v}=a O_{v}$ for some $a \in \mathfrak{m}_{v}$. For $i \geq 1$, the map $1+\mathfrak{m}_{v}^{i} \rightarrow \bar{F}_{v}$, $1+a^{i} b \mapsto \pi_{v}(b)$, is a group homomorphism with kernel $1+\mathfrak{m}_{v}^{i+1}$.

Lemma 7.1. Let $(E, u) /(F, v)$ be an extension of discrete valued fields with the same value group and residue field. Then:

(a) $\left(1+\mathfrak{m}_{u}^{i}\right) /\left(1+\mathfrak{m}_{v}^{i}\right) \cong E^{\times} / F^{\times}$canonically for all $i \geq 1$.

(b) For every $\mathscr{T}_{u^{-}}$-open subgroup $S$ of $E^{\times}$, one has $E^{\times}=F^{\times} S$. 
Proof. (a) For $i=1$ this follows from the exact sequences (5-1) and (5-2), with $F, S$ replaced by $E, F^{\times}$, respectively. For $1 \leq i$, the preceding remark gives a commutative diagram with exact rows:

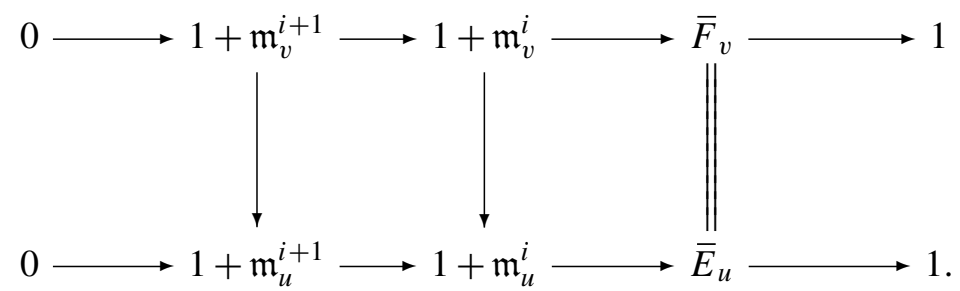

The snake lemma gives rise to a canonical isomorphism

$$
\left(1+\mathfrak{m}_{u}^{i+1}\right) /\left(1+\mathfrak{m}_{v}^{i+1}\right) \stackrel{\sim}{\longrightarrow}\left(1+\mathfrak{m}_{u}^{i}\right) /\left(1+\mathfrak{m}_{v}^{i}\right),
$$

so we are done by induction.

(b) Since $u$ is discrete, the subgroups $1+\mathfrak{m}_{u}^{i}, i=1,2,3, \ldots$, form a local basis for $\mathscr{T}_{u}$ at 1 . Hence there exists $i$ with $1+\mathfrak{m}_{u}^{i} \leq S$. By (a), $E^{\times}=F^{\times}\left(1+\mathfrak{m}_{u}^{i}\right)$, so $E^{\times}=F^{\times} S$.

Now let $p$ be a prime number and let $q=p^{d}$ be a $p$-power with $d \geq 1$.

Proposition 7.2. Let $v$ be a discrete valuation on a field $F$ such that $\operatorname{char} F=0$ and char $\bar{F}_{v}=p$. Let $(E, u)$ be the completion of $(F, v)$ and let $S=\left(F^{\times}\right)^{q}\left(1+q^{2} \mathfrak{m}_{v}\right)$. Then Res : $K_{*}^{M}(F) / S \rightarrow K_{*}^{M}(E) / q$ is an isomorphism.

Proof. By the Hensel-Rychlik lemma [Fesenko and Vostokov 1993, II (1.3), Corollary 2], $1+q^{2} \mathfrak{m}_{u} \leq\left(E^{\times}\right)^{q}$. In particular, $\left(E^{\times}\right)^{q}$ is $\mathscr{T}_{u}$-open in $E$. By Lemma 7.1(b), $E^{\times}=F^{\times}\left(1+q \mathfrak{m}_{u}\right)$. Hence $\left(E^{\times}\right)^{q}=\left(F^{\times}\right)^{q}\left(1+q^{2} \mathfrak{m}_{u}\right)$. It follows that $F \cap\left(E^{\times}\right)^{q}=\left(F^{\times}\right)^{q}\left(1+q^{2} \mathfrak{m}_{v}\right)=S$.

Since $F$ is $\mathscr{T}_{u}$-dense in $E$, the assertion now follows from Proposition 2.2.

Note that here the field $E$ is a complete, discrete valued field of characteristic 0 and finite residue field of characteristic $p$. Therefore it is a finite extension of $\mathbb{Q}_{p}$. For a detailed analysis of the Milnor $K$-ring of such fields, refer to [Fesenko and Vostokov 1993, Chapter IX].

The following theorem extends arguments of Pop, which are implicit in the proof of [Pop 1988, Korollar 2.7]. In Theorem 7.4 below we use it in conjunction with Theorem 6.2 to compute the functor $K_{*}^{M}(F) / S$ in another mixed characteristic situation.

Theorem 7.3. Let $v$ be a valuation of rank 1 on a field $F$ such that char $F=0$ and char $\bar{F}_{v}=p$. Suppose that $F^{\times} /\left(F^{\times}\right)^{p}\left(1+p \mathfrak{m}_{v}\right)$ is finite. Then either

(a) $v\left(F^{\times}\right)$is discrete and $\bar{F}_{v}$ is finite, or

(b) $v\left(F^{\times}\right)$is p-divisible and $\bar{F}_{v}$ is perfect. 
Proof. Let $S=\left(F^{\times}\right)^{p}\left(1+p \mathfrak{m}_{v}\right)$. We break the argument into five parts.

Part I: $\bar{F}_{v}$ is perfect. Indeed, $\bar{S}_{v}=\left(\bar{F}_{v}^{\times}\right)^{p}$. By the exact sequences (5-1) and (5-2), $\bar{F}_{v}^{\times} /\left(\bar{F}_{v}^{\times}\right)^{p}$ is finite. Since char $\bar{F}_{v}=p$, this quotient must be trivial [Efrat 2003, Corollary 1.6], as desired.

Part II: $S \cap G_{v}=G_{v}^{p}\left(1+p \mathfrak{m}_{v}\right)$. To show this, consider the commutative diagram of exponentiations by $p$ :

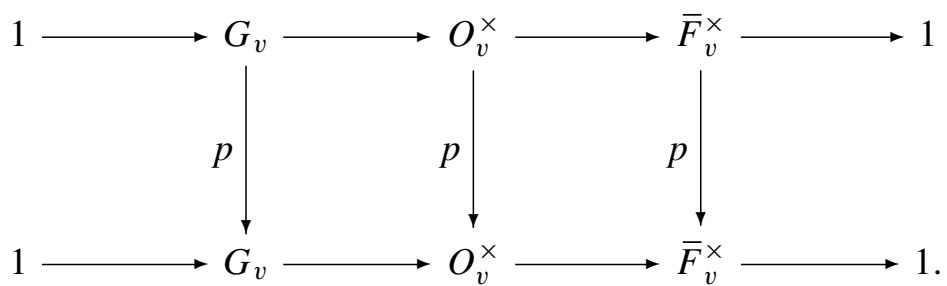

Since char $\bar{F}_{v}=p$, the right vertical map is injective. The snake lemma implies that $\left(O_{v}^{\times}\right)^{p} \cap G_{v}=G_{v}^{p}$. Hence also $\left(F^{\times}\right)^{p} \cap G_{v}=G_{v}^{p}$. Since $1+p \mathfrak{m}_{v} \leq G_{v}$ we obtain

$$
S \cap G_{v}=\left(\left(F^{\times}\right)^{p} \cap G_{v}\right)\left(1+p \mathfrak{m}_{v}\right)=G_{v}^{p}\left(1+p \mathfrak{m}_{v}\right) .
$$

Part III: $S \cap G_{v} \subseteq(1-S)\left(1+p \mathfrak{m}_{v}\right)$. Indeed, recall that $p \mid\left(\begin{array}{l}p \\ i\end{array}\right)$ for $i=1, \ldots, p-1$. Hence for every $a \in \mathfrak{m}_{v}$ we have

$$
(1-a)^{p} \in 1-a^{p}+p \mathfrak{m}_{v}=\left(1-a^{p}\right)\left(1+p \mathfrak{m}_{v}\right) \subseteq(1-S)\left(1+p \mathfrak{m}_{v}\right) .
$$

Thus $G_{v}^{p} \subseteq(1-S)\left(1+p \mathfrak{m}_{v}\right)$. Now use Part II.

Part IV: $v\left(F^{\times}\right)$is either discrete or p-divisible. In view of the structure of the ordered group $\mathbb{R}$, it suffices to find $0<\gamma \in v\left(F^{\times}\right)$such that for every $b \in F$ with $0<v(b)<\gamma$, one has $v(b) \in p v\left(F^{\times}\right)$. Since $F^{\times} / S$ is finite, the sequences (5-1) and (5-2) imply that $G_{v} /\left(S \cap G_{v}\right)$ is also finite. Hence we may take $\gamma$ as in Lemma 6.1. By property (i) of $W_{\gamma}$ and since $W_{\gamma} \leq G_{v}$, we have $1-b \in G_{v}=\left(S W_{\gamma}\right) \cap G_{v}=$ $\left(S \cap G_{v}\right) W_{\gamma}$. It therefore follows from part III and from property (ii) of $W_{\gamma}$ that $1-b \in(1-S) W_{\gamma}$. So choose $s \in S$ with $1-b \in(1-s) W_{\gamma}$. As $W_{\gamma} \leq G_{v}$ we get $1-s \in G_{v}$. Hence

$$
v(b-s)=v\left(\frac{b-s}{1-s}\right)=v\left(1-\frac{1-b}{1-s}\right) \geq \gamma .
$$

Since $v(b)<\gamma$, necessarily $v(b)=v(s) \in v(S)=p v\left(F^{\times}\right)$, as desired.

Part V: When $v\left(F^{\times}\right)$is discrete, $\bar{F}_{v}$ is finite. Indeed, as we have observed, in this case

$$
G_{v} /\left(1+\mathfrak{m}_{v}^{2}\right)=\left(1+\mathfrak{m}_{v}\right) /\left(1+\mathfrak{m}_{v}^{2}\right) \cong \bar{F}_{v}
$$


Using again that $p \mid\left(\begin{array}{c}p \\ i\end{array}\right)$ for $1 \leq i \leq p-1$, we get

$$
G_{v}^{p}\left(1+p \mathfrak{m}_{v}\right) \leq 1+\mathfrak{m}_{v}^{2} .
$$

In light of Part II, this gives rise to a group epimorphism $G_{v} /\left(S \cap G_{v}\right) \rightarrow \bar{F}_{v}$. We have already noted that $G_{v} /\left(S \cap G_{v}\right)$ is finite. Consequently so is $\bar{F}_{v}$.

Theorem 7.4. Let $v$ be a valuation of rank 1 on a field $F$ such that char $F=0$ and char $\bar{F}_{v}=p$. Let $S=\left(F^{\times}\right)^{q}\left(1+q^{2} \mathfrak{m}_{v}\right)$ and suppose that $\left(F^{\times}: S\right)<\infty$. Then one of the following holds:

(a) $v\left(F^{\times}\right)$is discrete, $\bar{F}_{v}$ is finite, and $K_{*}^{M}(F) / S \cong K_{*}^{M}(E) / q$ for the completion $E$ of $F$ with respect to $v$;

(b) $v\left(F^{\times}\right)$is $p$-divisible and $K_{r}^{M}(F) / S=0$ for all $r \geq 2$.

Proof. We have $v(S)=q v\left(F^{\times}\right)$and $\bar{S}_{v}=\left(\bar{F}_{v}^{\times}\right)^{q}$. Since

$$
\left(F^{\times}\right)^{q}\left(1+q^{2} \mathfrak{m}_{v}\right) \leq\left(F^{\times}\right)^{p}\left(1+p \mathfrak{m}_{v}\right),
$$

the finiteness assumption implies that $\left(F^{\times}:\left(F^{\times}\right)^{p}\left(1+p \mathfrak{m}_{v}\right)\right)<\infty$. By Theorem 7.3 , one of the following cases occurs:

Case (i): $v\left(F^{\times}\right)$is discrete and $\bar{F}_{v}$ is finite. Then we apply Proposition 7.2.

Case (ii): $v\left(F^{\times}\right)$is p-divisible and $\bar{F}_{v}$ is perfect. Then $v(S)=v\left(F^{\times}\right)$and $\bar{S}_{v}=$ $\bar{F}_{v}^{\times}$. The exact sequences (5-1) and (5-2) therefore show that $F^{\times}=S G_{v}$. Since $S$ is $\mathscr{T}_{v}$-open in $F$, Theorem 6.2 implies that $K_{r}^{M}(F) / S=0$ for $r \geq 2$.

\section{References}

[Arason et al. 1987] J. K. Arason, R. Elman, and B. Jacob, "Rigid elements, valuations, and realization of Witt rings", J. Algebra 110:2 (1987), 449-467. MR 89a:11041 Zbl 0629.10016

[Bass and Tate 1973] H. Bass and J. Tate, "The Milnor ring of a global field", pp. 349-446 in Algebraic K-theory, II: "Classical" algebraic K-theory and connections with arithmetic (Seattle, 1972), edited by H. Bass, Lecture Notes in Math. 342, Springer, Berlin, 1973. MR 56 \#449 Zbl 0299.12013

[Becker and Köpping 1977] E. Becker and E. Köpping, "Reduzierte quadratische Formen und Semiordnungen reeller Körper”, Abh. Math. Sem. Univ. Hamburg 46 (1977), 143-177. MR 58 \#21934 Zbl 0365.12011

[Bourbaki 1972] N. Bourbaki, Elements of mathematics. Commutative algebra, Hermann, Paris, 1972. MR 50 \#12997 Zbl 0279.13001

[Efrat 1999] I. Efrat, "Construction of valuations from K-theory", Math. Res. Lett. 6:3-4 (1999), 335-343. MR 2001i:12011 Zbl 0963.12003

[Efrat 2003] I. Efrat, "Demuškin fields with valuations", Math. Z. 243:2 (2003), 333-353. MR 2004d:11116 Zbl 01922384

[Fesenko and Vostokov 1993] I. B. Fesenko and S. V. Vostokov, Local fields and their extensions, Translations of Mathematical Monographs 121, American Mathematical Society, Providence, RI, 1993. MR 94d:11095 Zbl 0781.11042 
[Hwang and Jacob 1995] Y. S. Hwang and B. Jacob, "Brauer group analogues of results relating the Witt ring to valuations and Galois theory", Canad. J. Math. 47:3 (1995), 527-543. MR 97a:12004 Zbl 0857.12002

[Jacob 1981] B. Jacob, "On the structure of Pythagorean fields", J. Algebra 68:2 (1981), 247-267. MR 82g:12020 Zbl 0457.10007

[Kahn 1997] B. Kahn, "La conjecture de Milnor (d'après V. Voevodsky)", pp. 379-418, Exp. 834-5 in Sém. Bourbaki 1996/97, Astérisque 245, Soc. mat. de France, Paris, 1997. MR 2000a:19002 Zbl 0916.19001

[Lam 1983] T. Y. Lam, Orderings, valuations and quadratic forms, CBMS Regional Conference Series in Mathematics 52, American Mathematical Society, 1983. MR 85e:11024 Zbl 0516.12001 [Lang 1984] S. Lang, Algebra, 2nd ed., Addison-Wesley, Reading, MA, 1984. MR 86j:00003 Zbl 0712.00001

[Marshall 1980] M. Marshall, Abstract Witt rings, Queen's Papers in Pure and Applied Mathematics 57, Queen's University, Kingston, Ont., 1980. MR 84b:10032 Zbl 0451.10013

[Marshall 1996] M. A. Marshall, Spaces of orderings and abstract real spectra, Lecture Notes in Mathematics 1636, Springer, Berlin, 1996. MR 98b:14041 Zbl 0866.12001

[Matsumura 1980] H. Matsumura, Commutative algebra, Mathematics Lecture Note Series 56, Benjamin/Cummings, Reading, MA, 1980. MR 82i:13003 Zbl 0441.13001

[Milnor 1970] J. Milnor, "Algebraic K-theory and quadratic forms", Invent. Math. 9 (1970), 318344. MR 41 \#5465 Zbl 0199.55501

[Neukirch et al. 2000] J. Neukirch, A. Schmidt, and K. Wingberg, Cohomology of number fields, Grundlehren der Math. Wiss. 323, Springer, Berlin, 2000. MR 2000j:11168 Zbl 0948.11001

[Pop 1988] F. Pop, “Galoissche Kennzeichnung p-adisch abgeschlossener Körper”, J. Reine Angew. Math. 392 (1988), 145-175. MR 89k:12014 Zbl 0671.12005

[Wadsworth 1983] A. R. Wadsworth, " $p$-Henselian field: $K$-theory, Galois cohomology, and graded Witt rings", Pacific J. Math. 105:2 (1983), 473-496. MR 84m:12026 Zbl 0506.12024

[Ware 1981] R. Ware, "Valuation rings and rigid elements in fields", Canad. J. Math. 33:6 (1981), 1338-1355. MR 83i:10028 Zbl 0514.10015

Received August 29, 2004. Revised January 14, 2005.

\author{
IDO EFRAT \\ DEPARTMENT OF MATHEMATICS \\ BEN-GURION UNIVERSITY OF THE NEGEV \\ P.O. BOX 653 \\ BE'ER-SHEVA 84105 \\ ISRAEL \\ efrat@math.bgu.ac.il
}

